\title{
Influence of Electron-Electron Interaction on Electron Distributions in Short Si-MOSFETs Analysed Using the Local Iterative Monte Carlo Technique
}

\author{
T. MIETZNER ${ }^{\mathrm{a}, *}$, J. JAKUMEIT $^{\mathrm{b}}$ and U. RAVAIOLI ${ }^{\mathrm{c}}$ \\ aII. Phys. Inst., Universität Köln, Zülpicher Str. 77, 50937 Köln, Germany; ${ }^{\mathrm{b}}$ Institute for Algorithm and Scientific \\ Computing (SCAI), GMD-German National Research Center for Information Technology, Schloß Birlinghoven, \\ 53754 Sankt Augustin, Germany; ${ }^{\mathrm{c}}$ Beckman Institute and Department of Electrical and Computer Engineering, \\ University of Illinois at Urbana-Champaign, Urbana, IL 61801, USA
}

The effects of electron-electron interaction on the electron distribution in n-channel metal-oxide-semiconductor field-effect transistors (MOSFETs) are studied using the Local Iterative Monte Carlo (LIMO) technique. This work demonstrates that electronelectron scattering can be efficiently treated within this technique. The simulation results of a $90 \mathrm{~nm}$ Si-MOSFET are presented. We observe an increase of the high energy tail of the electron distribution at the transition from channel to drain.

Keywords: Monte Carlo; Device simulation; Electron-electron interaction; Local Iterative Monte Carlo

\section{INTRODUCTION}

New generations of MOSFETs are generally characterized by the presence of high electric fields and high energy electrons (hot electrons) that create reliability problems for the transistor. Hot carriers can get trapped in the oxide or create interface traps at the $\mathrm{Si} / \mathrm{SiO}_{2}$ interface. In short channel MOSFETs with bias voltages far below the $\mathrm{SiO}_{2}$ barrier height of $3.2 \mathrm{eV}$ electron-electron
(EE) interaction may still heat electrons to energies which allow a damage of the device. In this work the Local Iterative Monte Carlo (LIMO) technique $[1,2]$ is used as an numerical efficient tool to investigate the influence of $\mathrm{EE}$ interaction on the electron energy distribution. Besides the numerical efficiency LIMO gives the possibility to calculate EE scatterings rates selfconsistently using Fermis Golden rule, since the electron distribution is always accessible.

*Corresponding author. Tel.: +49 221 470-5766, Fax: +49221 470-2980, e-mail: tm@obelix.ph2.uni-koeln.de 


\section{LIMO TECHNIQUE}

The LIMO technique can be divided into two main parts, the short local Monte Carlo (MC) simulation steps and the iteration process. For the short MC steps, test particles are simulated in the same way as in a conventional MC-simulation for a short time of free flight. A number of such MC-steps are performed for each initial state of a discretization of the device region and energy space. In an iteration process the electron distribution in the whole device is calculated by exchanging local charge densities due to the information of the MC steps. It can be shown that the results converge towards a solution of the Boltzmann transport equation. Due to the piecewise nature of the LIMO technique the computation time is very efficiently distributed over phase space in contrast to standard MC algorithms, where the computational effort is proportional to the electron density if no statistical enhancement (or variance reduction) techniques are used. Therefore the LIMO technique is especially suited to the study of hot electron effects in semiconductor devices.

The LIMO algorithm used in this work is based on a full band structure model and the ensemble constant time technique for the time flight generation. The full band structure is calculated using the empirical pseudopotential model of Cohen and Bergstresser [3], with form factors taken from [4]. The scattering mechanisms include acoustic phonon scattering, $f$ - and $g$-type $X-X$ and $X-L$ optical phonon scattering, ionized impurity scattering (Ridley's model $[5,6]$ ), and impact ionization (Kane's model [7]).

The simulation of electron-electron (EE) scattering events can be treated differently in the LIMO technique compared to conventional MC programs, since the the electron distribution is always accessible. The scattering rate for shortrange EE process, which depends on the the electron distribution itself, can be self-consistently recalculated at any time during the iteration process. A special technique (Direct Exchange (DE) technique) has been developed for the treatment of the partner electron in a short-range EE events. The long-range part of the EE is treated as an additional inelastic scattering process since the LIMO approach does not include self-consistent solution of the Poisson equation. The electric field profile was calculated with the MOCA program from the University of Illinois and it remained unchanged during the simulation [8]. The LIMO approach might be able to show additional aspects of EE effects and, in this way, contribute to the still controversial discussion on the impact of EE on hot electron effects in modern short channel MOSFETs.

\section{ELECTRON-ELECTRON MODEL}

For the calculation of $\mathrm{EE}$ scattering rates the EE interaction has been split into two components. One component is associated with the motion of individual electrons. The other is associated with collective oszillation, the so-called plasmons. The separation occurs at roughly the Debye length [9]. The scattering rates for SEE (short-range electron-electron) and PEE (longrange electron - plasmon) scattering have been calculated by first order perturbation theory (Fermi's Golden Rule) [11, 12]. We have used the momentum relaxation scattering rate for description of the SEE and PEE interaction which simplifies the search for a final full band wave vector [10]. The SEE and PEE scattering has been incorporated in the same way as the other scattering mechanisms. Details of the model used will be published elsewhere.

Once a PEE event is stochastically determined during a MC step according to the momentum relaxation rate, the new state $\mathbf{k}^{\prime}$ can be chosen isotropy in $\mathbf{k}$-space with new energy $E(\mathbf{k}) \pm \hbar \omega_{p}[10]$.

The SEE scattering rate has been calculated as a function of the relative vector between the two involved electrons in the center of mass system. In order to obtain the SEE scattering rate as function of the investigated electron state $\mathbf{k}$ one has to 
integrate the scattering rate over all possible scattering partners, weighted by the electron distribution. Once a SEE scattering event occurs during a MC step we use a special procedure to handle the partner electron (see Fig. 1). In contrast to the MC electron, for which the SEE is just another scattering event during the short flight simulation, the electron density represented by the scattering partner is immediately exchanged in the electron distribution $f d$ in order to gain energy conservation. Therefore we call the method Direct Exchange (DE) technique. The scattering partner electron is chosen by a rejection technique that weights the scattering partner electrons by the momentary distribution of electrons. In order to avoid an overestimation of the SEE rate due to a double-count during a MC step, we have multiplied the SEE rate by a factor $1 / 2$ [14].

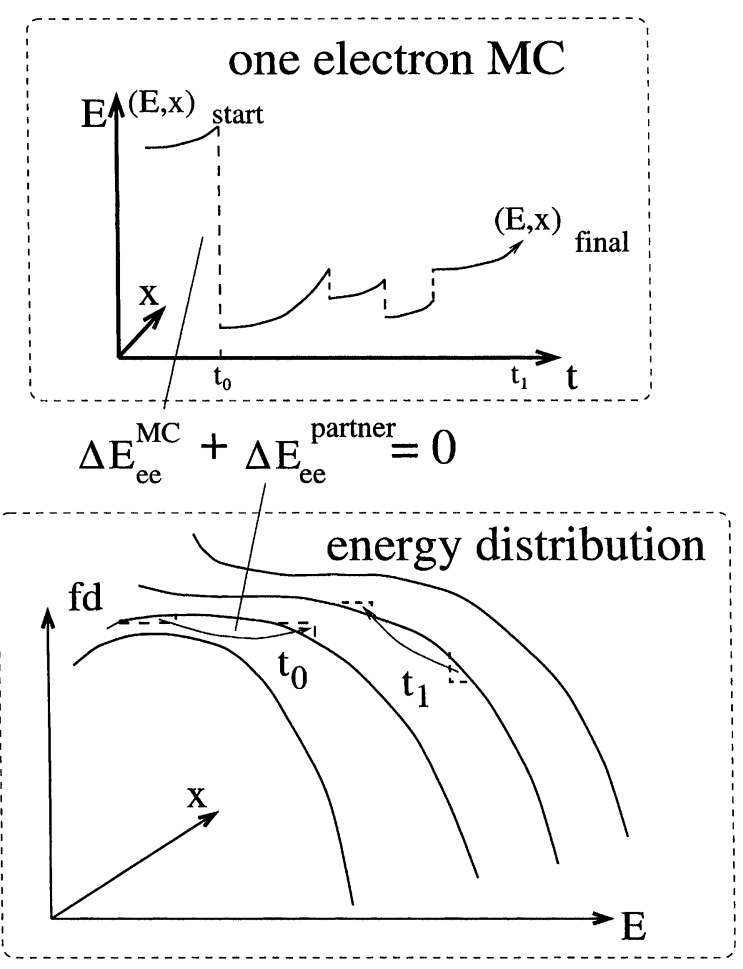

FIGURE 1 Schematic illustration of the DE technique. The electron distribution $f d$ is directly modified due to the energy change of the partner electrons involved in the short-range scattering event. Total energy $E_{\text {tot }}=E_{P}+E_{M C}$ and momentum have to be conserved.

\section{RESULTS}

The LIMO technique has been applied to 1-D simulations of a $90 \mathrm{~nm}$ n-channel Si-MOSFET. We refer to Ref. [15] for a detailed description of the device topology. In such short devices EE scattering may play an important role for hot electron phenomena like injection of electrons into gate oxide and parasitic bipolar action [8]. EE effects may heat electrons over the $\mathrm{SiO}_{2}$ barrier even if the voltage drop across the device is below $3.2 \mathrm{eV}$.

Figures 2 and 3 show the electron distributions in a Si-MOSFET with an effective channel length of $90 \mathrm{~nm}$ for drain and gate bias values of $1.5 \mathrm{~V}$ and $3 \mathrm{~V}$, respectively. Four distributions are plotted in the figure showing, from cold to hot distributions, the results obtained with only PEE, no EE scattering, the full model (PEE + SEE) and only SEE. The transition from channel to drain is at $200 \mathrm{~nm}$. PEE acts as an additional energy relaxation channel and leads to a colder distribution over the whole energy range. SEE has no effect at low energies but yields a significant increase of the high energy tail by pushing the electron distributions to be more Fermi like. Both effects increase inside the drain region (starting at $200 \mathrm{~nm}$ ) with increasing carrier density, but the increase is larger

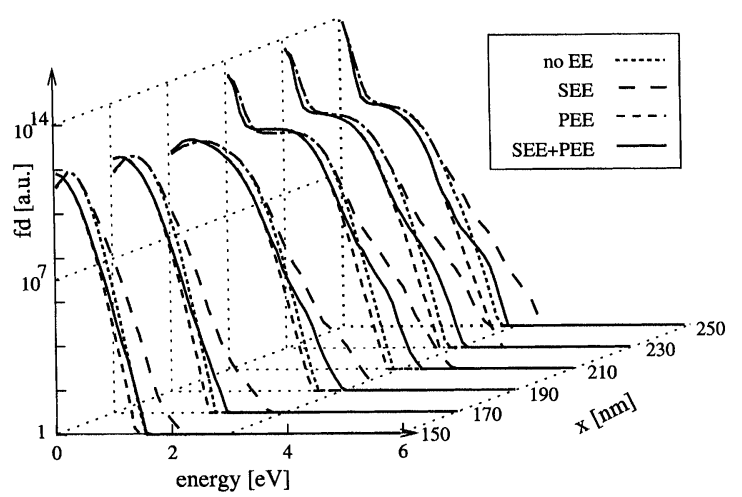

FIGURE 2 Influence of EE scattering on the energy distribution of electrons in a $90 \mathrm{~nm}$ n-channel Si-MOSFET for $V_{g}=V_{d}=1.5 \mathrm{~V}$. The interesting region at the transition from channel to drain is plotted. The transition from channel to drain is at approximately $200 \mathrm{~nm}$. 


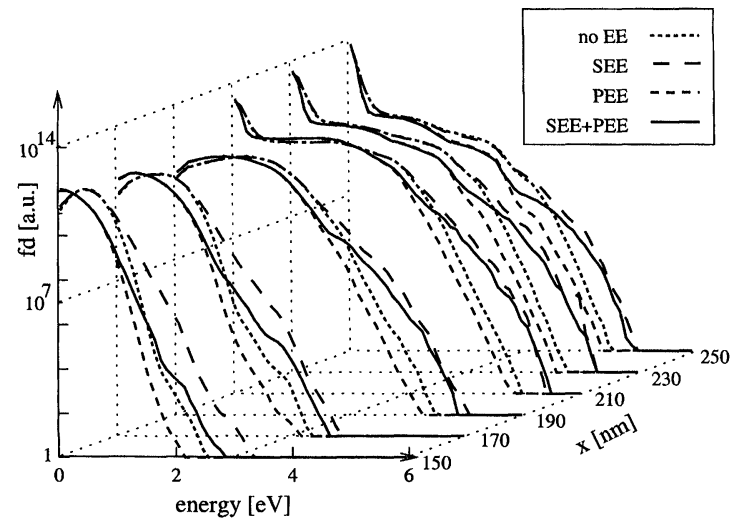

FIGURE 3 Same as Figure 2 for $V_{g}=V_{d}=3 \mathrm{~V}$.

for the SEE contribution. Three effects increase SEE scattering at the transition from channel to drain. Beside the increase of the electron density, the electron temperature has its maximum (about $8000 \mathrm{~K}$ ), leading to a lower screening, and the distribution is far from a Fermi like behavior. At $1.5 \mathrm{~V}$ at drain and gate the tail of the distribution is significantly increased just at the $\mathrm{SiO}_{2}$ barrier energy of $3.2 \mathrm{eV}$. This should be important for the gate current and related hot electron degradation phenomena.

\section{CONCLUSIONS}

A special full-band MC technique, the LIMO technique, has been used to study the influence of electron-electron interaction on electron distributions in $\mathrm{n}$-channel Si-MOSFETs. Since the electron distribution is always accessible in the LIMO technique, the long-range plasmon as well as the short-range direct interaction could be calculated using Fermi's Golden rule. The scattering rates were updated during the simulation to achieve a self-consistent calculation. To handle the second partner in a direct interaction event the Direct Exchange (DE) technique was developed.
An 1-D model was then used to investigate the influence of electron-electron scattering on the energy distributions in n-channel Si-MOSFETs. Our results show that electron-electron (EE) scattering can lead to significant increase of the high energy tail due to short-range EE scattering. For a $90 \mathrm{~nm}$ MOSFET with $1.5 \mathrm{~V}$ at drain and gate, SEE leads to a increase of the distribution just at the $\mathrm{SiO}_{2}$ barrier energy of $3.2 \mathrm{eV}$, which may have a significant effect on hot electron degradation phenomena.

\section{Acknowledgments}

This work was partially supported by the Semiconductor Research Corporation, contract 98-SJ406, by the National Center for Computational Electronics (NCCE) through National Science Foundation grant ECS 95-26127, and the Deutsche Forschungsgemeinschaft GK Scientific Computing.

\section{References}

[1] Jakumeit, J., Sontowski, T. and Ravaioli, U., In: Proc. 6th International Workshop of Computational Electronics, Osaka, 1998.

[2] Jakumeit, J. and Ravaioli, U., submitted to IEEE Trans. ED.

[3] Cohen, M. L. and Bergstresser, T. K. (1996). Phys. Rev., 141, 789.

[4] Chelikowsky, J. R. and Cohen, M. L. (1976). Phys. Rev., 14, 556.

[5] Ridley, B. K. (1977). J. Phys. C, 10, 1589.

[6] Van de Roer, T. G. and Widdershoven, F. P. (1986). J. Appl. Phys., 59, 813.

[7] Kane, E. O. (1967). Phys. Rev., 159(3), 624.

[8] Duncan, A., Ravaioli, U. and Jakumeit, J. (1998). IEEE Trans. Elec. Dev., 45(4).

[9] Pines, D. and Bohm, D. (1952). Physical Review, 85(2), 338.

[10] Kosina, H. (1999). IEEE Trans. Elec. Dev., 46(6), 1196.

[11] Singh, J. (1996). Physics of Semiconductors and Their Heterostructures, McGraw-Hill.

[12] Ridley, B. K. (1988). Quantum Processes in Semiconductors. Oxford Science Publications, Clarendon Press.

[13] Jacoboni, C. and Reggiani, L. (1983). Reviews of Modern Physics, 55(3), 645.

[14] Fischetti, M. V., Laux, S. E. and Crabbé, E. (1995). J. Appl. Phys., 78(2), 1058.

[15] see http://www.mtl.mit.edu:80/Well 

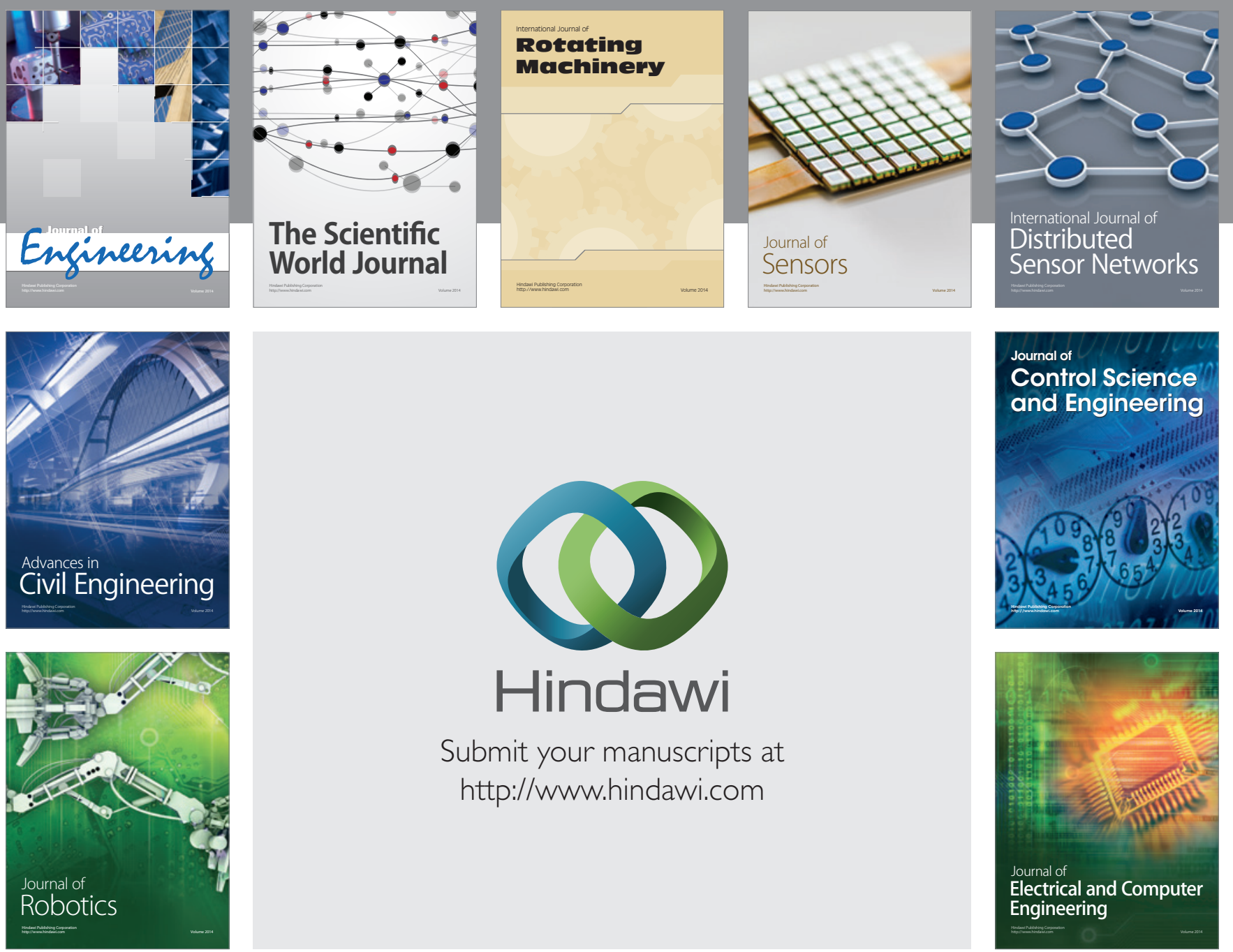

Submit your manuscripts at

http://www.hindawi.com
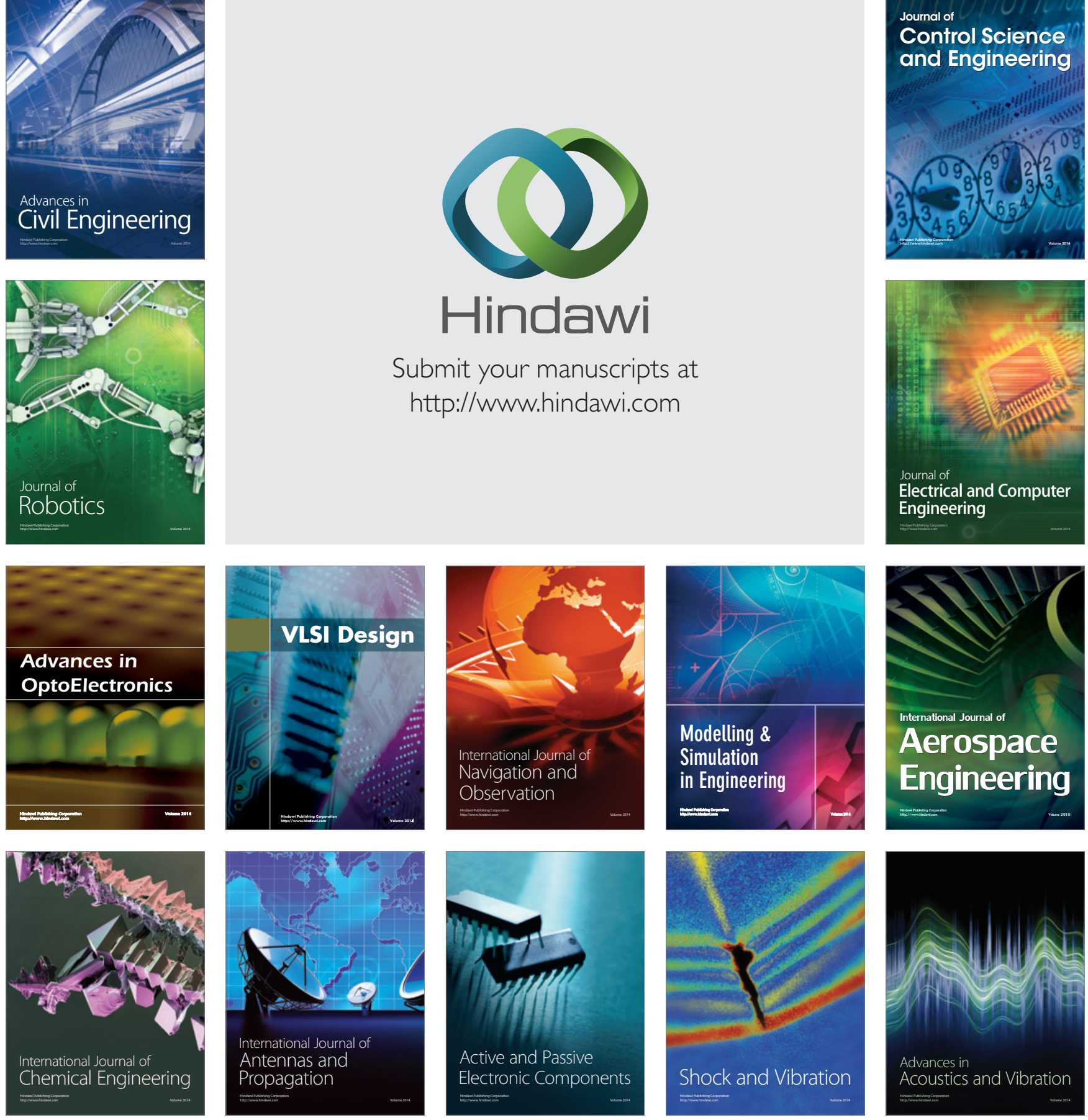